ARTIGO ORIGINAL / ORIGINAL ARTICLE

\title{
Coexistência de comportamentos de risco à saúde e o contexto familiar entre adolescentes brasileiros, Pesquisa Nacional de Saúde do Escolar (2015)
}

\section{Co-occurrence of health risk behaviors and the family context among. Brazilian adolescents, National Survey of School Health (2015)}

\author{
Roberta Mendes Abreu Silval (D), Amanda Cristina de Souza Andrade ${ }^{\| \prime, I I}$ (D), \\ Waleska Teixeira Caiaffa"II (D), Vanessa Moraes Bezerral (D)
}

RESUMO: Objetivo: Avaliar a associação entre a coexistência de comportamentos de risco (CR) e o contexto familiar em adolescentes brasileiros. Métodos: Estudo transversal com 101.534 estudantes do $9^{\circ}$ ano do ensino fundamental na Pesquisa Nacional de Saúde do Escolar 2015. A coexistência de CR foi estimada pelo somatório da presença de comportamento sedentário, baixo consumo de frutas e consumo regular de álcool e tabaco. A prevalência foi avaliada usando o diagrama de Venn e a análise multivariada por meio da regressão logística ordinal de chances parciais. Resultados: $8,8 \%$ dos adolescentes não apresentaram CR; 34,5\% tinham um; $42,7 \%$ tinham dois; e $14,1 \%$, três ou quatro. As combinações mais prevalentes foram entre comportamento sedentário e baixo consumo de frutas (33,8\%); e comportamento sedentário com baixo consumo de frutas e consumo regular de álcool (9,5\%). Tiveram maior chance de coexistência aqueles que tinham mães com maiores escolaridades em todos os modelos, não morar com os pais [0, 1 e 2 vs. 3 : (odds ratio - OR = 1,21; intervalo de confiança de $95 \%$ - IC95\% 1,07 - 1,37)], ter pais que às vezes, nunca ou raramente entendiam seus problemas e preocupações [0, 1 e 2 vs. 3: (OR = 1,62; IC95\% 1,49 - 1,76)] e acompanhavam o dever de casa [0, 1 e 2 vs. 3: (OR = 1,77; IC95\% 1,62 - 1,93)]; e realizar refeições com os pais ou responsáveis < 4 dias / semana para os três modelos. Conclusão: Os CR relacionados à saúde tendem a se agrupar entre os adolescentes brasileiros e estão associados a características do contexto familiar. Esses achados apontam para a necessidade de ações de promoção de saúde com foco na simultaneidade, e não de forma isolada.

Palavras-chave: Adolescente. Comportamentos relacionados com a saúde. Fatores de risco. Saúde do adolescente.

IInstituto Multidisciplinar em Saúde, Universidade Federal da Bahia - Vitória da Conquista (BA), Brasil.

"Universidade Federal de Mato Grosso - Cuiabá (MT), Brasil.

I'Faculdade de Medicina, Universidade Federal de Minas Gerais - Belo Horizonte (MG), Brasil.

Autora correspondente: Roberta Mendes Abreu Silva. Rua Hormindo Barros, 58, Candeias, CEP: 45029-094, Vitória da Conquista, BA, Brasil. E-mail: roberta.m.abreu@hotmail.com

Conflito de interesses: nada a declarar - Fonte de financiamento: nenhuma. 
ABSTRACT: Objective: To evaluate the association between the co-occurrence of risk behaviors (RB) and the family context in Brazilian adolescents. Methods: Cross-sectional study on 101,534 students from the $9^{\text {th }}$ grade of elementary school in the National Survey of School Health - PeNSE 2015. The co-occurrence of RB was estimated by the sum of the presence of sedentary behavior, low fruit consumption, regular consumption of alcohol and tobacco. Prevalence was assessed using the Venn diagram and multivariate analysis by the ordinal logistic regression model of partial proportional odds. Results: $8.8 \%$ of the adolescents did not have RB; $34.5 \%$ had one; $42.7 \%$ had two; and $14.1 \%$, three or four. The most prevalent combinations were between sedentary behavior and low fruit consumption (33.8\%); sedentary behavior with low fruit consumption and regular consumption of alcohol (9.5\%). Those who were more likely to present co-occurrence had mothers with higher education level in all models, did not live with their fathers $[0,1$ and 2 vs. $3:(\mathrm{OR}=1.21 ; 95 \% \mathrm{CI} 1.07-1.37)]$, had parents who sometimes, never or rarely understood their problems and concerns $[0,1$ and 2 vs. 3 : $(\mathrm{OR}=1.62 ; 95 \% \mathrm{CI} 1.49-1.76)]$ and monitored their homework [0, 1 and 2 vs. 3 : $(\mathrm{OR}=1.77 ; 95 \% \mathrm{CI} 1.62-1.93)]$; and had meals with parents or guardians $<4$ days / week for the three models. Conclusion: Health RB tend to cluster among Brazilian adolescents and are related to characteristics of the family context. These findings point to the need for health promotion actions focusing on simultaneity and not in isolation.

Keywords: Adolescent. Health behavior. Risk factors. Adolescent health.

\section{INTRODUÇÃO}

Os comportamentos de risco à saúde são apontados como precursores de condições futuras de adoecimento e desenvolvimento de diversas comorbidades, entre elas as doenças crônicas não transmissíveis (DCNT) $)^{1,2}$. A Organização Mundial da Saúde (OMS) indica que os principais comportamentos de risco relacionados às DCNT são o consumo regular de tabaco e álcool, a inatividade física, o comportamento sedentário e a dieta inadequada ${ }^{2}$.

A adoção de comportamentos de risco na adolescência está ligada à busca por maior independência, identidade e aceitação nos grupos sociais dos quais os adolescentes fazem parte ${ }^{1}$. Logo, compreender os efeitos da coexistência dos comportamentos de risco é importante, pois eles repercutem sobre o processo saúde-doença ao longo da adolescência e na vida adulta ${ }^{2,3}$.

Estudos mostram que os comportamentos de saúde ou de risco para a saúde iniciados na adolescência estão inter-relacionados a múltiplos determinantes dos âmbitos biológico, individual, social e contextual $1^{1-3}$. Quanto ao âmbito contextual, os aspectos familiares recebem destaque, pois o contexto familiar ocupa papel importante no microssistema de desenvolvimento humano. Esse contexto é responsável por funções de apoio individual, proteção e afeto ao adolescente, proporcionando um ambiente propício à aprendizagem, ao amadurecimento e ao exercício da cidadania ${ }^{4}$.

O contexto familiar pode ter efeito sobre o desenvolvimento das potencialidades durante a adolescência e como o adolescente se relaciona com o meio social no qual está inserido. Dessa forma, as vulnerabilidades e os problemas vivenciados nas relações familiares, os comportamentos adotados no núcleo familiar, a fragilidade dos vínculos familiares, a falta de 
apoio ao processo da autonomia e de monitoramento parental são fatores importantes que podem estar ligados ao desenvolvimento de comportamentos de risco em adolescentes ${ }^{5,6}$.

Algumas pesquisas têm mostrado como os comportamentos de risco podem coexistir no cotidiano dos adolescentes ${ }^{7.9}$. Sabe-se que os fatores determinantes para ocorrência de um comportamento de risco tendem a fomentar a ocorrência simultânea de outros ${ }^{8,9}$. Cada comportamento de risco pode apresentar um efeito distinto para a saúde, e diferentes comportamentos de risco podem refletir de forma sinérgica para proporcionar o adoecimento ${ }^{8}$. Na literatura poucos estudos discutem de maneira analítica a coexistência de comportamentos de risco ou de proteção à saúde entre os adolescentes ${ }^{2,8,10,11}$, sobretudo a relação deles com as características do contexto familiar ${ }^{5,11}$.

Logo, o objetivo do trabalho foi avaliar a associação entre o contexto familiar e a coexistência de comportamentos de risco para a saúde entre os adolescentes brasileiros, por meio da Pesquisa Nacional de Saúde do Escolar (PeNSE) 2015.

\section{MÉTODOS}

Trata-se de um estudo populacional baseado em dados secundários obtidos por uma pesquisa transversal com escolares matriculados no $9^{\circ}$ ano do ensino fundamental que frequentavam escolas públicas e privadas no Brasil. Foram utilizados dados da terceira edição da PeNSE, que é representativa das cinco regiões do Brasil ${ }^{12}$. Os microdados, relacionados ao banco 1 da PeNSE, estão disponibilizados na página eletrônica do Instituto Brasileiro de Geografia e Estatística. A PeNSE foi aprovada pela Comissão Nacional de Ética em Pesquisa (Conep) com parecer $\mathrm{n}^{\circ}$ 1.006.467, de 30 de março de 2015.

\section{CÁLCULO AMOSTRAL}

A amostra de escolas da PeNSE foi selecionada de 53 estratos geográficos. Desse total, 27 estratos correspondiam aos 26 municípios das capitais e ao Distrito Federal, e os 26 estratos restantes, a alguns municípios, fora das capitais, representando cada uma das unidades da federação. A seleção ocorreu em três estágios:

- municípios ou grupos de municípios $(\mathrm{n}=675)$;

- escolas $(\mathrm{n}=3.160)$;

- $\operatorname{turmas}(\mathrm{n}=4.159)^{12}$.

O cruzamento entre os estratos geográficos e a dependência administrativa da escola (privadas ou públicas) foi considerado para definir o tamanho da amostra, de acordo com o número de turmas do $9^{\circ}$ ano. As escolas formam unidades primárias de amostragem, e as turmas, as unidades secundárias. O sorteio das unidades primárias de amostragem dentro de cada estrato foi realizado por meio de amostragem sistemática com probabilidade proporcional ao número de escolas nos estratos. O total de participantes foi de 101.534 estudantes que tinham idade maior ou igual a 13 anos ${ }^{12}$. 
Todos os alunos presentes no dia da coleta, nas turmas sorteadas, foram convidados a participar da pesquisa. As respostas foram registradas diretamente pelos escolares em questionário eletrônico. Mais informações sobre o cálculo amostral e a determinação do tamanho da amostra estão disponíveis no estudo de Oliveira et al. ${ }^{13}$.

\section{VARIÁVEIS ESTUDADAS}

\section{Variável dependente}

A variável dependente foi composta de quatro comportamentos relacionados à saúde: comportamento sedentário, consumo regular de álcool, consumo regular de tabaco e consumo de frutas. Dessa forma, a coexistência do comportamento de risco está relacionada à ocorrência simultânea de no mínimo dois e no máximo quatro desses comportamentos avaliados. Foram classificados como comportamento sedentário aqueles escolares que relataram ficar sentados mais que duas horas por $\mathrm{dia}^{9,14}$, no contraturno escolar; como consumo regular de álcool os que consumiram pelo menos uma dose de bebida alcoólica nos últimos 30 dias; como consumo regular de tabaco os que tinham fumado em pelo menos um dia nos últimos 30 dias; e como consumo irregular de frutas aqueles que consumiam frutas quatro vezes ou menos por semana ${ }^{15}$.

Os quatro comportamentos de risco (CR) estudados foram codificados individualmente em uma variável binária (presença do $C R=1$; ausência do $C R=0$ ). A coexistência de $C R$ foi estimada por meio do somatório desses quatro comportamentos, sendo ranqueada de zero (nenhum) até quatro CR simultâneos.

\section{Variáveis independentes}

Variáveis relacionadas ao contexto familiar abordadas pela PeNSE ${ }^{12}$ foram consideradas como explicativas principais. São elas: escolaridade materna (nenhuma, ensino fundamental incompleto, ensino fundamental completo e ensino médio incompleto, ensino médio completo e ensino superior incompleto, ensino superior completo); estrutura familiar (mora com pai e mãe, mora com o pai ou com a mãe, não mora com os pais); pais que entendiam os problemas e as preocupações (frequentemente ou sempre, às vezes, nunca ou raramente); pais que acompanhavam o dever de casa (frequentemente ou sempre, às vezes, nunca ou raramente); e realizar refeições com os pais (cinco dias ou mais por semana, até quatro dias por semana).

\section{Covariáveis}

As covariáveis foram sexo, idade e nível econômico, por meio do escore de bens e serviços (EBS), utilizadas como fatores de ajuste. Para a criação do EBS, os seguintes 
itens foram usados: posse de telefone fixo, telefone celular, computador, internet, automóvel e motocicleta, presença de banheiro dentro de casa e presença de empregada doméstica três ou mais vezes por semana. Cada item apresentado anteriormente recebeu um peso, que corresponde ao inverso da frequência de posse ou presença no total da amostra estudada. O escore total de cada aluno foi obtido somando-se os pesos dos respectivos itens e categorizando-os em terços da distribuição observada na amostra ${ }^{16}$.

\section{TRATAMENTO DE DADOS FALTANTES}

Do total de alunos respondentes da PeNSE 2015, 25\% ( $\mathrm{n}=25.434)$ não soube informar a escolaridade da mãe. Dessa forma, foi implementado um processo de imputação múltipla de dados que compreendeu a identificação de variáveis preditoras: sexo, bens familiares (carro, telefone fixo, telefone celular, número de banheiros em casa) e serviços (empregada doméstica e acesso à internet em casa $)^{17}$. A variável escolaridade paterna não foi incluída na análise, pois esta não foi mensurada na PeNSE 2015. Foi utilizado o método Multiple Imputation by Chained Equations (MICE) para atribuir valores numéricos à variável escolaridade materna ${ }^{18}$.

\section{ANÁLISE DOS DADOS}

A prevalência dos múltiplos CR foi estimada pelo somatório dos comportamentos. Foi utilizado o diagrama de Venn para a representação gráfica da coexistência de CR para a população estudada. Trata-se de um método usado para comparar e visualizar conjuntos de dados de diversos grupos que consiste em intersecções gráficas, que representam os diferentes grupos de conjuntos de dados. A prevalência da sobreposição e separação entre as formas gráficas permite uma representação visual das relações entre os diferentes CR analisados ${ }^{19}$.

Para a descrição das variáveis, utilizaram-se a distribuição de frequências e seus respectivos intervalos de confiança. O número de $\mathrm{CR}$ associados ao contexto familiar foi avaliado por meio de análise bivariada, e os dados foram apresentados com suas respectivas proporções para cada categoria do desfecho.

Foi realizada a análise de regressão logística ordinal de chances proporcionais parciais para identificar as variáveis do contexto familiar associadas ao número de CR. Esse modelo foi adotado, uma vez que foi violada a hipótese de chances proporcionais, também chamada de suposição de regressão paralela ${ }^{20}$. Foram estimados as odds ratio (OR) ajustadas e seus intervalos de confiança de 95\% (IC95\%). Todas as análises foram feitas no software estatístico Stata 12.0, e a complexidade do desenho amostral foi considerada em todas as análises. 
Tabela 1. Características sociodemográficas e proporção da coexistência de comportamento de risco em relação às variáveis de contexto familiar, para adolescentes brasileiros, Pesquisa Nacional de Saúde do Escolar (PeNSE) 2015.

\begin{tabular}{|c|c|c|c|c|c|c|c|}
\hline \multirow{2}{*}{ Variáveis } & \multirow{2}{*}{$\%$} & \multirow{2}{*}{ IC95\% } & \multicolumn{5}{|c|}{ Coexistência de comportamentos de risco } \\
\hline & & & 0 & 1 & 2 & 3 ou 4 & Valor $p^{*}$ \\
\hline \multicolumn{8}{|l|}{ Sexo } \\
\hline Masculino & 48,7 & $48,1-49,3$ & 48,3 & 50,4 & 48,8 & 44,3 & \multirow{2}{*}{$<0,001$} \\
\hline Feminino & 51,3 & $50,7-51,9$ & 51,7 & 49,6 & 51,2 & 55,7 & \\
\hline \multicolumn{8}{|l|}{ Idade (anos) } \\
\hline 13 & 17,9 & $16,9-18,9$ & 17,8 & 18,4 & 19,4 & 12,8 & \multirow{4}{*}{$<0,001$} \\
\hline 14 & 51,2 & $50,3-52,1$ & 50,2 & 51,5 & 53,1 & 46,0 & \\
\hline 15 & 19,9 & $19,1-20,6$ & 21,1 & 18,9 & 18,3 & 25,6 & \\
\hline $16-19$ & 11,0 & $10,4-11,6$ & 10,9 & 11,2 & 9,2 & 15,6 & \\
\hline \multicolumn{8}{|l|}{ Nível econômico } \\
\hline $1^{\circ}$ tercil & 32,4 & $31,4-33,4$ & 37,5 & 36,5 & 29,3 & 28,1 & \multirow{3}{*}{$<0,001$} \\
\hline $2^{\circ}$ tercil & 36,6 & $35,8-37,4$ & 34,7 & 35,0 & 38,2 & 37,7 & \\
\hline $3^{\circ}$ tercil & 31,0 & $29,8-32,1$ & 27,8 & 28,5 & 32,5 & 34,2 & \\
\hline \multicolumn{8}{|l|}{ Escolaridade materna } \\
\hline Sem estudo & 7,7 & $7,3-8,1$ & 9,8 & 9,0 & 6,5 & 6,6 & \multirow{5}{*}{$<0,001$} \\
\hline Fundamental incompleto & 26,7 & $25,9-27,4$ & 28,0 & 27,9 & 25,5 & 26,5 & \\
\hline $\begin{array}{l}\text { Fundamental completo/Médio } \\
\text { incompleto }\end{array}$ & 17,2 & $16,6-17,8$ & 17,0 & 17,4 & 17,3 & 16,9 & \\
\hline $\begin{array}{l}\text { Médio completo/Superior } \\
\text { incompleto }\end{array}$ & 30,6 & $29,9-31,3$ & 28,7 & 28,5 & 32 & 32,7 & \\
\hline Superior completo & 17,8 & $16,8-18,7$ & 16,5 & 17,2 & 18,7 & 17,3 & \\
\hline \multicolumn{8}{|l|}{ Estrutura familiar } \\
\hline Mora com o pai e a mãe & 59,3 & $58,5-60$ & 62,4 & 62,1 & 59,2 & 51 & \multirow{3}{*}{$<0,001$} \\
\hline Mora com o pai ou a mãe & 35 & $34,3-35,7$ & 31,9 & 32,3 & 35,5 & 42 & \\
\hline Não mora com os pais & 5,7 & $5,5-6$ & 5,7 & 5,6 & 5,3 & 7 & \\
\hline \multicolumn{8}{|l|}{ Pais que entendem problemas* } \\
\hline Frequentemente/sempre & 43,8 & $43,1-44,4$ & 59,2 & 49 & 40,6 & 31 & \multirow{3}{*}{$<0,001$} \\
\hline Às vezes & 22,8 & $22,3-23,3$ & 19,9 & 22,3 & 23,9 & 22,6 & \\
\hline Nunca/raramente & 33,4 & $32,8-34,1$ & 20,9 & 28,7 & 35,5 & 46,4 & \\
\hline \multicolumn{8}{|c|}{ Pais que acompanham as atividades de casa* } \\
\hline Frequentemente/sempre & 31,8 & $31,2-32,5$ & 52,7 & 31,1 & 27 & 20,3 & \multirow{3}{*}{$<0,001$} \\
\hline Às vezes & 23,8 & $23,3-24,2$ & 20,6 & 24,4 & 24,9 & 20,7 & \\
\hline Nunca/raramente & 44,4 & $43,7-45,1$ & 26,7 & 38,5 & 48,1 & 59 & \\
\hline \multicolumn{8}{|c|}{ Refeição com os pais ou responsáveis* } \\
\hline Regularmente ( $\geq 5$ dias/semana) & 74 & $73,4-74,7$ & 83,5 & 78,5 & 72,1 & 63 & \multirow{2}{*}{$<0,001$} \\
\hline$\leq 4$ dias por semana & 26 & $25,3-26,6$ & 16,5 & 21,5 & 27,9 & 37 & \\
\hline
\end{tabular}

*Teste de Pearson; \%: frequência da população estudada; IC95\%: intervalo de confiança de 95\%. 


\section{RESULTADOS}

Entre o total de participantes, a maioria era do sexo feminino, 51,2\% tinha 14 anos e $36,6 \%$ pertencia ao segundo tercil do nível econômico. Para o contexto familiar, $30,6 \%$ tinha mães com ensino médio completo ou ensino superior incompleto, a maioria dos adolescentes morava com o pai e a mãe, $43,8 \%$ tinha pais que entendiam seus problemas frequentemente ou sempre, $44,4 \%$ tinha pais que nunca ou raramente acompanhavam as atividades de casa e $74 \%$ fazia refeições com os pais regularmente (Tabela 1).

O aumento das proporções de coexistência de três ou quatro CR foi observado para os adolescentes do sexo feminino, com idade de 15 anos ou mais, que moravam com o pai ou a mãe ou não moravam com os pais e para os adolescentes cujas mães tinham ensino médio completo ou superior incompleto. Verificou-se, ainda, aumento na proporção de coexistência de CR para os adolescentes pertencentes aos segundo e terceiro tercis econômicos, que os pais nunca ou raramente entendiam os problemas e as preocupações, que nunca ou raramente acompanhavam as lições de casa e entre aqueles que realizavam refeições com os pais ou responsáveis menos de cinco dias por semana (Tabela 1).

Em relação à coexistência de CR relacionados à saúde, 8,8\% (IC95\% 8,5 - 9,2) dos adolescentes não a apresentaram; 34,5\% (IC95\% 33,8 - 35,1) tinham um CR; 42,7\% (IC95\% 42,0 - 43,2) tinham dois; e 14,1\% (IC95\% 13,7 - 14,6), três ou quatro.

O CR mais frequente entre os adolescentes foi o comportamento sedentário $(68,1 \%)$, seguido do baixo consumo de frutas $(67,2 \%)$, consumo regular de álcool $(23,4 \%)$ e, por último, consumo de tabaco (5,6\%). Conforme diagrama de Venn, as interseções mais frequentes entre os CR foram entre comportamento sedentário e baixo consumo de frutas $(33,8 \%)$ e entre comportamento sedentário, baixo consumo de frutas e consumo regular de álcool (9,5\%) (Figura 1).

$\mathrm{Na}$ análise multivariada, os adolescentes que relataram ter mães com mais escolaridade tiveram maior chance de coexistência de CR para os três modelos de comparação [0 vs. 1 , 2 e 3: $(\mathrm{OR}=1,29$; IC95\% 1,10 - 1,52)]; [0 e 1 vs. 2 e 3: $(\mathrm{OR}=1,31$; IC95\% 1,18 - 1,45)]; [0, 1 e 2 vs. 3: (OR = 1,19; IC95\% 1,03-1,37)] quando comparados aos que tinham mães sem escolaridade. Morar com um dos pais em comparação a morar com ambos foi positivamente associado à chance de coexistência de CR nos três modelos analisados [0 vs. 1, 2 e 3: $(\mathrm{OR}=1,09 ;$ IC95\% 1,01 - 1,19)]; [0 e 1 vs. 2 e 3: (OR = 1,21; IC95\% 1,15-1,27)]; [0, 1 e 2 vs. $3:(\mathrm{OR}=1,36$; IC95\% 1,26-1,46)] e a categoria não morar com os pais apenas para a comparação 0,1 e 2 vs. 3 ou mais CR (OR = 1,21; IC95\% 1,07 - 1,37) (Tabela 2).

Foi associado a maiores chances de coexistência de CR ter pais que às vezes, nunca ou raramente entendiam seus problemas e preocupações [0, 1 e 2 vs. 3 : (OR = 1,62; IC95\% $1,49-1,76)]$, assim como ter pais que acompanhavam nunca ou raramente o dever de casa [0, 1 e 2 vs. 3: (OR = 1,77; IC95\% 1,62 - 1,93)]. Realizar refeições com os pais ou responsáveis de maneira irregular foi positivamente associado à coexistência de CR em todos os modelos avaliados quando comparado àqueles que realizam regularmente as refeições $[0$ vs. 1, 2 e 3: (OR = 1,44; IC95\% 1,29-1,61)]; [0 e 1 vs. 2 e 3: (OR = 1,39; IC95\% 1,31-1,47)]; [0, 1 e 2 vs. 3: $(\mathrm{OR}=1,43$; IC95\% 1,33-1,54)] (Tabela 2). 


\section{DISCUSSÃO}

Este estudo encontrou associações significativas entre a coexistência de CR à saúde e variáveis de contexto familiar para os adolescentes brasileiros. A chance de apresentar maior número de CR foi maior para os adolescentes que relataram: ter mães com mais escolaridade; morar com o pai ou a mãe, ou não morar com os pais; ter pais que às vezes, nunca ou raramente entendiam seus problemas e preocupações e acompanhavam o dever de casa; e realizar refeições com os pais ou responsáveis quatro dias ou menos por semana.

O consumo de tabaco e álcool, o baixo consumo de frutas e legumes e o comportamento sedentário são alguns dos comportamentos relacionados ao desenvolvimento de DCNT e

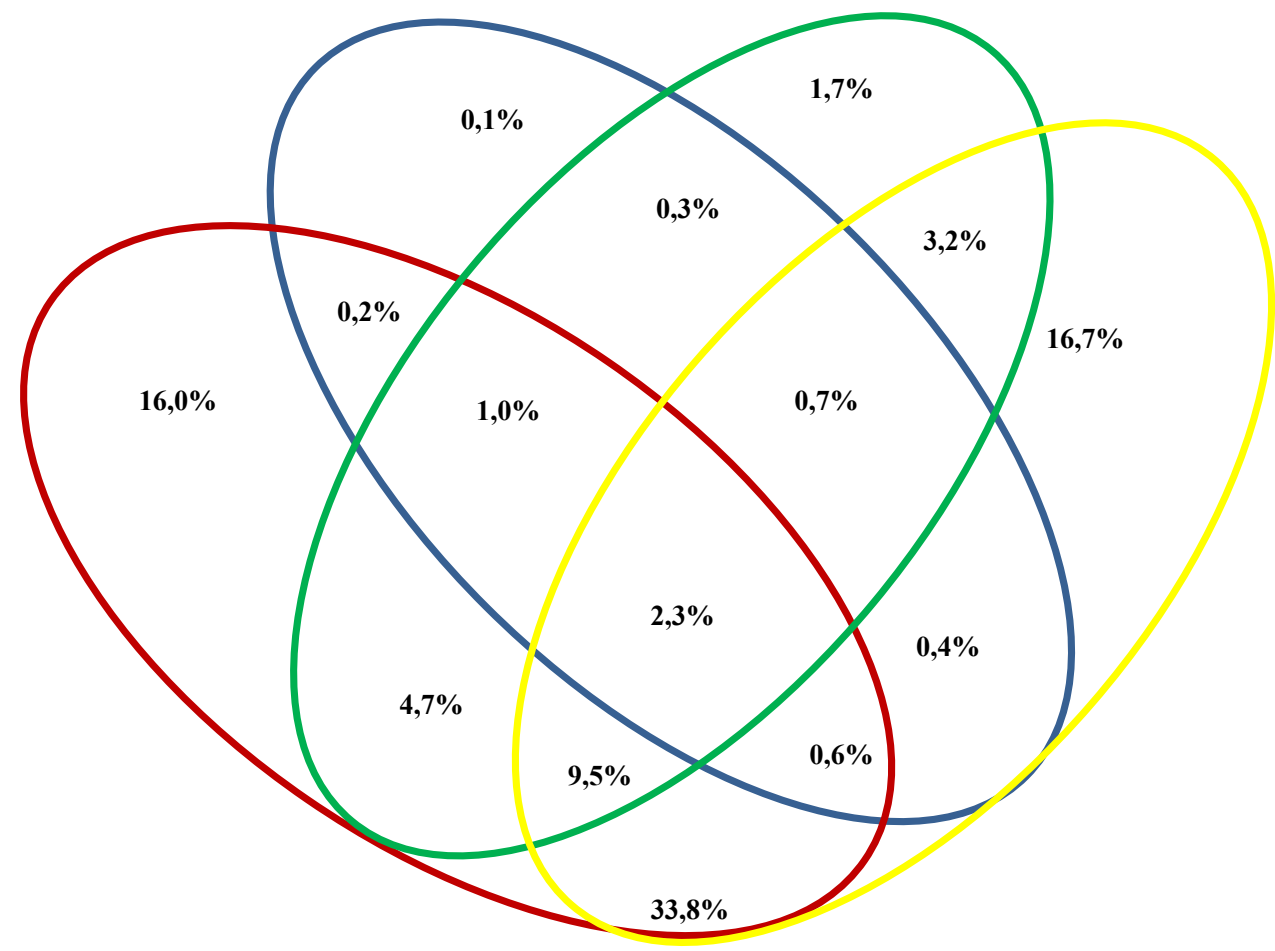

\footnotetext{
Comportamento sedentário $(68,1 \%)$

Consumo regular de tabaco $(5,6 \%)$

Consumo regular de álcool $(23,4 \%)$

Baixo consumo de frutas $(67,2 \%)$
}

$8,8 \%$

Figura 1. Coexistência de comportamentos de risco em adolescentes brasileiros, Pesquisa Nacional de Saúde do Escolar (PeNSE) 2015. 
ao aumento de mortalidade para a população mundial ${ }^{1,2}$. A OMS reconhece o papel da soma dos riscos nos níveis primário e secundário de atenção à saúde e indica que o risco global para o adoecimento é aumentado quando múltiplos CR estão presentes².

Tabela 2. Análise multivariada de chances parciais para coexistência de comportamentos de risco e contexto familiar em adolescentes brasileiros, Pesquisa Nacional de Saúde do Escolar (PeNSE) 2015.

\begin{tabular}{|c|c|c|c|c|c|c|}
\hline \multirow{2}{*}{ Variáveis } & \multicolumn{4}{|c}{ Comparações } \\
\cline { 2 - 6 } & $\begin{array}{c}\text { O versus 1, 2 e 3 } \\
\text { ou mais }\end{array}$ & $\begin{array}{c}\text { O e 1 versus 2 e 3 } \\
\text { ou mais }\end{array}$ & $\begin{array}{c}\text { 0, } 1 \text { e } 2 \text { versus } 3 \\
\text { ou mais }\end{array}$ \\
\cline { 2 - 7 } & OR & IC95\% & OR & IC95\% & OR & IC 95\%
\end{tabular}

Contexto familiar*

Escolaridade materna

\begin{tabular}{|c|c|c|c|c|c|c|}
\hline Sem estudo & 1 & & 1 & - & 1 & - \\
\hline Fundamental incompleto & 1,22 & $1,06-1,40$ & 1,25 & $1,14-1,37$ & 1,25 & $1,10-1,42$ \\
\hline $\begin{array}{l}\text { Fundamental completo/ } \\
\text { Médio incompleto }\end{array}$ & 1,29 & $1,10-1,50$ & 1,30 & $1,18-1,44$ & 1,22 & $1,06-1,41$ \\
\hline $\begin{array}{l}\text { Médio completo/Superior } \\
\text { incompleto }\end{array}$ & 1,33 & $1,15-1,54$ & 1,44 & $1,31-1,58$ & 1,35 & $1,19-1,54$ \\
\hline Superior completo & 1,29 & $1,10-1,52$ & 1,31 & $1,18-1,45$ & 1,19 & $1,03-1,37$ \\
\hline
\end{tabular}

Estrutura familiar

\begin{tabular}{|l|c|c|c|c|c|c|}
\hline Mora com o pai e a mãe & 1 & - & 1 & - & 1 & - \\
\hline Mora com o pai ou a mãe & 1,09 & $1,01-1,19$ & 1,21 & $1,15-1,27$ & 1,36 & $1,26-1,46$ \\
\hline Não mora com os pais & 0,94 & $0,80-1,11$ & 1,01 & $0,92-1,11$ & 1,21 & $1,07-1,37$ \\
\hline
\end{tabular}

Pais que entendem problemas

\begin{tabular}{l|c|c|c|c|c|c}
\hline Frequentemente/sempre & 1 & - & 1 & - & 1 & - \\
\hline Às vezes & 1,35 & $1,22-1,50$ & 1,27 & $1,20-1,35$ & 1,28 & $1,17-1,41$ \\
\hline Nunca/raramente & 1,58 & $1,43-1,76$ & 1,45 & $1,37-1,54$ & 1,62 & $1,49-1,76$ \\
\hline
\end{tabular}

Pais que acompanham o dever de casa

\begin{tabular}{l|c|c|c|c|c|c}
\hline Frequentemente/sempre & 1 & - & 1 & - & 1 & - \\
\hline Às vezes & 1,87 & $1,69-2,07$ & 1,48 & $1,39-1,58$ & 1,29 & $1,16-1,43$ \\
\hline Nunca/raramente & 2,43 & $2,20-2,68$ & 1,82 & $1,72-1,93$ & 1,77 & $1,62-1,93$ \\
\hline
\end{tabular}

\section{Refeição com os pais ou responsáveis}

\begin{tabular}{l|c|c|c|c|c|c} 
Regularmente $(\geq 5$ dias/semana) & 1 & - & 1 & - & 1 & - \\
\hline$\leq 4$ dias por semana & 1,44 & $1,29-1,61$ & 1,39 & $1,31-1,47$ & 1,43 & $1,33-1,54$
\end{tabular}

*Ajustado para as variáveis sociodemográficas (sexo, idade e nível econômico); OR: odds ratio; IC95\%: intervalo de confiança de 95\%. 
Entre os comportamentos analisados, observou-se que os CR, como o comportamento sedentário e o consumo alimentar inadequado, podem coexistir, e a alta prevalência dessa coexistência foi associada a fatores sociodemográficos, familiares e comportamentais ${ }^{10,21}$. Além disso, a coexistência dos comportamentos analisados corrobora os achados de uma pesquisa que investigou adolescentes de 89 países para determinar os padrões de agrupamento. A coexistência de tabagismo, consumo de álcool, comportamento sedentário e baixo consumo de frutas e vegetais foi 6,81 vezes maior no sexo feminino e 2,51 vezes maior no masculino do que era esperado globalmente ${ }^{1}$.

A adoção de CR pode estar ligada a diversos fatores. Entre eles, podem-se destacar os contextos social e familiar no qual esses adolescentes estão inseridos ${ }^{6,12,22}$. Estudo que entrevistou adolescentes baseado na teoria da influência triádica categorizou os determinantes por meio dos contextos social, cultural, intrapessoal e identificou que o consumo de tabaco e o de álcool foram fortemente associados com as práticas parentais, CR de amigos e pais ${ }^{6}$. Além disso, pesquisa longitudinal demonstrou que crianças expostas a contextos sociofamiliares de vulnerabilidade e violência tendem a apresentar ansiedade, depressão, comorbidades e consumo de álcool durante a vida ${ }^{3}$. Dessa forma, é possível compreender que os contextos social e familiar podem compor um cenário de maior vulnerabilidade e estímulo à adoção de múltiplos $\mathrm{CR}$ à saúde ${ }^{6,12}$.

Os adolescentes que relataram ter mães com mais escolaridade apresentaram maiores chances de coexistência de CR para os três modelos analisados. Mais escolaridade materna pode representar também maior nível econômico, o que pode determinar maior poder de compra familiar, proporcionando, por exemplo, o acesso a jogos, aparelhos eletrônicos e alimentos não saudáveis ${ }^{10}$. Uma das possíveis explicações reside no fato de que níveis de escolaridade mais elevados representam mães que provavelmente trabalham fora de seus domicílios e, por conseguinte, tendem a ter menor controle sobre o que os adolescentes fazem no seu tempo livre ${ }^{23}$.

A escolaridade, a ocupação materna e a presença de CR podem ter inter-relações que perpassam pela estrutura familiar. Para a estrutura familiar, observou-se que os adolescentes que responderam morar com o pai ou a mãe ou não morar com os pais foram positivamente associados à coexistência de CR. Pais que compõem o núcleo monoparental tendem a estar divididos entre o sustento da casa, a sobrecarga de papéis e o suprimento da demanda de atenção e as necessidades emocionais e sociais que o adolescente requer ${ }^{24}$. Ademais, adolescentes que não moram com os pais podem estar sujeitos a menos regras e limites, tendo relação mais permissiva por parte de seus responsáveis ${ }^{25}$.

É importante ressaltar que transições na estrutura familiar, que vão do modelo nuclear intacto (composto de um casal de adultos e seus filhos socialmente reconhecidos) ${ }^{26}$ para a família uniparental ou monoparental, a reconstituída ou recasada (quando um ou ambos os cônjuges têm filho/filhos de um vínculo anterior) ${ }^{26}$, podem favorecer a presença de CR em adolescentes. Famílias com núcleos intactos tendem a apresentar contextos de maior estabilidade para os filhos quando comparadas a núcleos familiares reconstituídos ou monoparentais ${ }^{27,28}$. Adolescentes vindos de famílias em que houve divórcio tendem a ter uma avaliação negativa de seus pais, menor percepção de segurança pessoal e autocontrole quando comparados aos provenientes de famílias intactas ${ }^{6,29}$, entretanto deve-se levar em consideração que as relações no núcleo familiar estão interligadas às capacidades de plasticidade e de adequação dos indivíduos ${ }^{30}$. 
Foi associado a maiores chances de coexistência de CR ter pais que às vezes, nunca ou raramente entendiam os problemas e as preocupações. Essa associação pode ser explicada pela possibilidade de existir menor capacidade familiar para enfrentar e discutir, de forma democrática e madura, os problemas de seus filhos. O contexto familiar em que existe um distanciamento na relação entre os pais e os filhos parece não promover um ambiente que favoreça o diálogo ${ }^{29,31}$. Além disso, ter boa comunicação com os pais reflete na maneira como o adolescente expressa sua satisfação com a vida e à saúde . $^{\circ}$

Os escolares que responderam ter pais que acompanhavam o dever de casa às vezes, nunca ou raramente foram associados à coexistência de CR. Sabe-se que menor suporte familiar para o adolescente pode refletir em dificuldades que são vivenciadas e percebidas nas interações familiares. A variação do suporte familiar interfere nas percepções de autonomia e independência versus rejeição, indiferença, superproteção ou controle, podendo acarretar o aumento da possibilidade de adoção de $\mathrm{CR}^{27,32}$. A literatura aponta para a importância do acompanhamento parental, de forma que ter pais que se interessam pelas atividades cotidianas dos seus filhos e se relacionam com elas e sabem o que os filhos fazem no tempo livre influencia no CR na adolescência e pode constituir um fator protetor de condutas de risco nessa fase da vida ${ }^{31}$.

Os adolescentes que relataram realizar refeições com os pais ou responsáveis com menor frequência tiveram maiores chances de coexistência de CR. Um estudo anterior sugeriu que adolescentes que têm o hábito de realizar as refeições com os pais ou responsáveis estão associados a mais momentos de interação entre os membros da família e ao maior consumo de alimentos saudáveis ${ }^{33}$. Ademais, realizar refeições com os pais representa características de convivência e coesão familiar, que podem exercer efeito protetor para diversos CR. Quando os adolescentes possuem relação positiva com os pais e vivenciam boa convivência no contexto familiar, é esperada redução da adoção de $\mathrm{CR}^{31}$.

Identificar os CR e sua associação com o contexto familiar se torna importante, pois propicia o desenvolvimento de estratégias para o enfrentamento dos problemas e agravos, como a prevenção de DCNT, além de contribuir para o aprimoramento da política pública de promoção à saúde ${ }^{34}$.

Entretanto, por ser este um estudo transversal, não é possível inferir na natureza temporal. As comparações relacionadas à coexistência de CR devem ser realizadas com cautela, visto que as pesquisas apresentam múltiplos métodos, diversas definições e seleções de CR. A investigação de somente quatro CR pode ter limitado os resultados, contudo os comportamentos investigados estão entre os principais fatores de risco para as DCNT.

Todos os comportamentos estudados foram obtidos por meio do autorrelato dos adolescentes, $\mathrm{O}$ que pode levar à subestimação ou superestimação da prevalência, no entanto o autorrelato tem sido comumente usado em inquéritos nacionais, e a PeNSE integra um sistema de monitoramento da saúde do escolar que utiliza perguntas validadas e comparáveis a outros estudos internacionais ${ }^{12,35}$.

Quanto à variável estrutura familiar, a pergunta refere-se a morar com o pai ou morar com a mãe, o que pode limitar a caracterização dos diferentes arranjos familiares possíveis. A variável não permite captar as mudanças ocorridas nas formas de organização familiar, como estruturas familiares por casais do mesmo sexo e escolares que moram alternadamente em dois lares, por exemplo.

Por fim, entende-se que os CR estudados são potencialmente modificáveis, e o presente manuscrito identifica, para além das variáveis sociodemográficas descritas em estudos anteriores, a importância e o efeito do contexto familiar sobre a adoção de múltiplos CR nessa população. 


\section{REFERÊNCIAS}

1. Uddin R, Lee EY, Khan SR, Tremblay MS, Khan A. Clustering of lifestyle risk factors for non-communicable diseases in 304,779 adolescents from 89 countries: A global perspective. Prev Med. 2020; 131: 105955. https: / / doi.org/10.1016/j.ypmed.2019.105955

2. World Health Organization. Global health risks: mortality and burden of disease attributable to selected major risks. Genebra: World Health Organization; 2009.

3. Skinner ML, Hong S, Herrenkohl TI, Brown EC, Lee JO, Jung H. Longitudinal Effects of Early Childhood Maltreatment on Co-Occurring Substance Misuse and Mental Health Problems in Adulthood: The Role of Adolescent Alcohol Use and Depression. J Stud Alcohol Drugs 2016; 77(3): 464-72. https: / / doi.org/10.15288/ jsad.2016.77.464

4. Zappe JG, Dell'Aglio DD. Risco e Proteção no Desenvolvimento de Adolescentes que Vivem em Diferentes Contextos: Família e Institucionalização. Rev Colomb Psicol 2016; 25(2): 289-305. https: / / doi. org/10.15446/rcp.v25n2.51256

5. Velderman MK, Dusseldorp E, Nieuwenhuijzen MV, Junger M, Paulussen TG, Reijneveld SA. Cultural, social and intrapersonal factors associated with clusters of co-occurring health-related behaviours among adolescents. Eur J Public Health 2015; 25(1): 31-7. https: / / doi.org/10.1093/eurpub/cku051

6. Tomé G, Camacho I, Matos MG, Simões C. Influência da família e amigos no bem-estar e comportamentos de risco: Modelo explicativo. Psicol Saúde Doenças 2015; 16(1): 23-34. https:// doi.org/10.15309/15psd160104

7. Meader N, King K, Moe-Byrne T, Wright K, Graham H, Petticrew $\mathrm{M}$, et al. A systematic review on the clustering and co-occurrence of multiple risk behaviours. BMC Public Health 2016; 16: 657. https: / / doi.org/10.1186/ s12889-016-3373-6

8. Ricardo CZ, Azeredo CM, Machado de Rezende LF, Levy RB. Co-occurrence and clustering of the four major non-communicable disease risk factors in Brazilian adolescents: Analysis of a national schoolbased survey. PloS One 2019; 14(7): e0219370. https: / / doi.org/10.1371/journal.pone.0219370

9. Silva RMA, Bezerra VM, Medeiros DS. Experimentação de tabaco e fatores associados entre adolescentes da zona rural de Vitória da Conquista, BA, Brasil. Ciênc Saúde Coletiva 2019; 24(2): 431-41. https:// doi.org/10.1590/1413-81232018242.02962017

10. Ferreira NL, Claro RM, Mingoti SA, Lopes ACS. Coexistence of risk behaviors for being overweight among Brazilian adolescents. Prev Med 2017; 100: 135-42. https: / / doi.org/10.1016/j.ypmed.2017.04.018
11. Marques ES, Azeredo CM, de Oliveira A. Co-occurrence of Health Risk Behaviors Among Brazilian Adolescent Victims of Family Violence. J Interpers Violence 2018. https: / / doi.org/10.1177/0886260518786493

12. Instituto Brasileiro de Geografia e Estatística. Pesquisa Nacional de Saúde do Escolar: 2015. Rio de Janeiro: Instituto Brasileiro de Geografia e Estatística; 2016.

13. Oliveira MM, Campos MO, Andreazzi MAR, Malta DC. Características da Pesquisa Nacional de Saúde do Escolar-PeNSE. Epidemiol Serv Saúde 2017; 26(3): 605 16. https: / / doi.org/10.5123/s1679-49742017000300017

14. Canadian Society for Exercise Physiology. Canadian 24-Hour Movement Guidelines for Children and Youth (ages 5-17 years): An Integration of Physical Activity, Sedentary Behavior and Sleep. Canadian Society for Exercise Physiology; 2016.

15. Brasil. Ministério da Saúde. Secretaria de Atenção à Saúde. Departamento de Atenção Básica. Guia alimentar para a população brasileira. $2^{\mathrm{a}} \mathrm{ed}$. Brasil: Ministério da Saúde; 2014.

16. Levy RB, Castro IRR, Cardoso LO, Tavares LF, Sardinha LMV, Gomes FS, et al. Consumo e comportamento alimentar entre adolescentes brasileiros: Pesquisa Nacional de Saúde do Escolar (PeNSE), 2009. Ciênc Saúde Coletiva 2010; 15(Supl. 2): 3085-97. https:// doi.org/10.1590/S1413-81232010000800013

17. Azeredo CM, de Rezende LF, Canella DS, Moreira Claro R, de Castro IR, Luiz OC, et al. Dietary intake of Brazilian adolescents. Public Health Nutr 2015; 18(7): 1215-24. https://doi.org/10.1017/ S1368980014001463

18. Royston P, White IR. Multiple Imputation by Chained Equations (MICE): Implementation in Stata. J Stat Software 2011; 45(4). https: / / doi.org/10.18637/jss. v045.i04

19. Cai H, Chen H, Yi T, Daimon CM, Boyle JP, Peers C, et al. VennPlex-A Novel Venn Diagram Program for Comparing and Visualizing Datasets with Differentially Regulated Datapoints. Plos One 2013; 8(1): e53388. https: / / doi.org/10.1371/journal.pone.0053388

20. Abreu MNS, Siqueira AL, Caiaffa WT. Regressão logística ordinal em estudos epidemiológicos. Rev Saúde Pública 2009; 43(1): 183-94. https: / / doi.org/10.1590/ S0034-89102009000100025

21. Filippidis FT, Agaku IT, Vardavas CI. Geographic variation and socio-demographic determinants of the co-occurrence of risky health behaviours in 27 European Union member states. J Public Health (Oxf) 2016; 38(2): e13-20. https://doi.org/10.1093/ pubmed/fdv061 
22. Wikle JS, Hoagland A. Adolescent interactions with family and emotions during interactions: Variation by family structure. J Fam Psychol 2020; 34(5): 544-54. https: / / doi.org/10.1037/ fam0000625

23. Brito ALS, Hardman CM, Barros MVG. Prevalência e fatores associados à simultaneidade de comportamentos de risco à saúde em adolescentes. Rev Paul Pediatr 2015; 33(4): 423-30. https://doi.org/10.1016/j. rpped.2015.02.002

24. Melo SCH, Marin AH. Influência das composições familiares monoparentais no desenvolvimento da criança: revisão de literatura. Rev SPAGESP 2016; 17(1): 4-13.

25. Gomide PIC. Pais presentes, pais ausentes: regras e limites. Campinas: Vozes; 2017.

26. Encyclopædia Britannica. Nuclear family [Internet]. Encyclopædia britannica; 2015 [acessado em 2 set. 2019]. Disponível em: https: / www.britannica.com/ topic/nuclear-family

27. Baptista MN, Baptista ASD, Dias RR. Estrutura e suporte familiar como fatores de risco na depressão de adolescentes. Psicol Ciênc Prof 2001; 21(2): 52-61. https://doi.org/10.1590/ S1414-98932001000200007

28. Oliveira WA, Silva JL, Sampaio JMC, Silva MAI. Saúde do escolar: uma revisão integrativa sobre família e bullying. Ciênc Saúde Coletiva 2017; 22(5); 1553-64. https://doi. org/10.1590/1413-81232017225.09802015

29. Mombelli MA, Costa JB, Marcon SS, Moura CB. Family pillar and structure as risk factors to child stress. Estud Psicol (Campinas) 2011; 28(3): 327-35. https:/ / doi. org/10.1590/S0103-166X2011000300004

30. Dessen MA, Polonia AC. Family and school as context for human development. Paidéia (Ribeirão Preto) 2007; 17(36): 21-32. https: / / doi.org/10.1590/ S0103-863X2007000100003
31. Malta DC, Porto DL, Melo FCM, Monteiro RA, Sardinha LMV, Lessa BH. Family and the protection from use of tobacco, alcohol, and drugs in adolescents, National School Health Survey. Rev Bras Epidemiol 2011; 14(Supl. 1): 16677. https: / / doi.org/10.1590/S1415-790X2011000500017

32. Paiva FS, Ronzani TM. Estilos parentais e consumo de drogas entre adolescentes: revisão sistemática. Psicol Estud 2009; 14(1): 177-83. https:// doi.org/10.1590/ S1413-73722009000100021

33. Chan JC, Sobal J. Family meals and body weight. Analysis of multiple family members in family units. Appetite 2011; 57(2): 517-24. https:/ / doi.org/10.1016/j. appet.2011.07.001

34. Malta DC, Morais Neto OL, Silva MMA, Rocha D, Castro AM, Reis AAC, et al. Política Nacional de Promoção da Saúde (PNPS): capítulos de uma caminhada ainda em construção. Ciênc Saúde Coletiva 2016; 21(6): 1683-94. https: / / doi.org/10.1590/1413-81232015216.07572016

35. Tavares LF, Castro IRR, Cardoso LO, Levy RB, Claro $\mathrm{RM}$, Oliveira AF. Validade de indicadores de atividade física e comportamento sedentário da Pesquisa Nacional de Saúde do Escolar entre adolescentes do Rio de Janeiro, Brasil. Cad Saúde Pública 2014; 30(9): 186174. https: / / doi.org/10.1590/0102-311X00151913

\section{Recebido em: 05/08/2020 \\ Revisado em: 20/11/2020 \\ Aceito em: 15/12/2020}

Contribuição dos autores: Os autores Silva RMA, Bezerra VM, Andrade ACS e Caiaffa WT participaram da redação do artigo, análise dos dados, interpretação dos resultados e análise crítica relevante do conteúdo intelectual. Além das contribuições já citadas, todos os autores foram responsáveis por todos os aspectos do trabalho, na garantia de exatidão e integridade de qualquer parte da obra. Todos os autores leram e aprovaram a versão final deste manuscrito. 